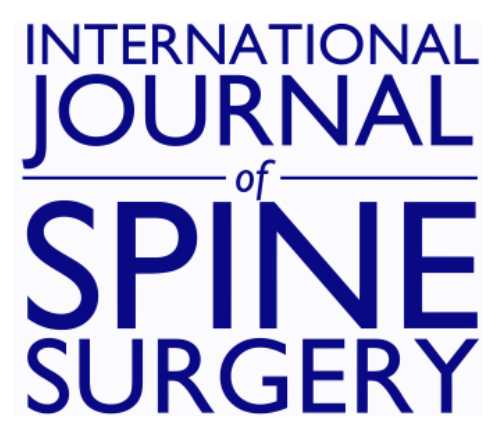

\title{
Hemivertebra Resection and Spinal Arthrodesis by Single-Stage Posterior Approach in Congenital Scoliosis and Kyphoscoliosis: Results at 9.6 Years Mean Follow-up
}

Marco Crostelli, Osvaldo Mazza, Massimo Mariani, Dario Mascello, Federico Tundo and Carlo Iorio

Int J Spine Surg published online 17 February 2022

http://ijssurgery.com/content/early/2022/02/17/8188

This information is current as of April 25, 2023.

Email Alerts Receive free email-alerts when new articles cite this article. Sign up at:

http://ijssurgery.com/alerts

The International Journal of Spine Surgery

2397 Waterbury Circle, Suite 1,

Aurora, IL 60504, Phone: +1-630-375-1432 


\title{
Hemivertebra Resection and Spinal Arthrodesis by Single-Stage Posterior Approach in Congenital Scoliosis and Kyphoscoliosis: Results at 9.6 Years Mean Follow-up
}

\author{
MARCO CROSTELLI, MD ${ }^{1}$; OSVALDO MAZZA, MD ${ }^{1}$; MASSIMO MARIANI, MD ${ }^{1}$; DARIO MASCELLO, MD ${ }^{1}$; \\ FEDERICO TUNDO, $\mathrm{MD}^{1}$; AND CARLO IORIO, $\mathrm{MD}^{1}$ \\ ${ }^{1}$ Spine Surgery Unit - Bambino Gesù Children's Hospital, Rome, Italy
}

\begin{abstract}
Background: Congenital kyphoscoliosis due to hemivertebra is generally treated surgically because of high risk of curve progression and high risk of nervous system complications. Modern posterior access surgical techniques, including total hemivertebra resection, can completely correct deformity without additional anterior access surgeries. The purpose of this study was to evaluate midterm results of hemivertebra resection and spinal arthrodesis; the hypothesis was that it is a safe, effective, and reproducible procedure.

Materials and Methods: From 2006 to 2019, hemivertebra resection and instrumented spinal arthrodesis with pedicle screws was performed on 82 patients with congenital vertebral deformities (62 scoliosis and 20 kyphoscoliosis) by posterior approach. Mean age at surgery was 8.6 years, and 22 patients were under 10 years of age. After stabilization patients have been braced for a period from 3 to 5 months.

Results: Mean follow-up was 9.6 years (range 1.2-12.8 years); mean kyphosis curve after surgery was reduced to $20^{\circ}$ Cobb; and mean scoliosis curve was reduced to $11^{\circ}$ Cobb. We experienced no major complications (postsurgical infection, instrumentation failure, severe neurological impairment, severe blood loss) at latest follow-up .

Conclusion: We strongly advocate one-time posterior hemivertebra resection and arthrodesis as the most suitable surgical procedure for congenital scoliosis due to hemivertebra. Posterior approach interventions with pedicle screws instrumentation are less invasive than combined anterior-posterior approach interventions. We think that posterior approach procedures can lead to excellent deformity correction in both frontal and sagittal views, optimal stability, and low risk of nervous injury.

Clinical Relevance: Congenital scoliosis treatment is one of the most challeging conditions a spine surgeon has to face. We advocate that a one-stage posterior approach for hemivertebrectomy and fusion is a reliable, safe tachnique, whom excellent results remain stable at a mid/long-term follow-up.
\end{abstract}

Level of Evidence: $\quad$ Level 4.

Other \& Special Categories

Keywords: congenital scoliosis, hemivertebra resection, posterior vertebral arthrodesis

\section{INTRODUCTION}

Segmentation and formation defects in vertebrae cause congenital deformities of the spine: scoliosis, kyphosis, and kyphoscoliosis. Hemivertebrae are the most frequent cases of congenital deformity, and their growth potential is similar to a normal vertebra, creating a wedge-shaped deformity on both the sagittal and frontal planes, which can progress during spine growth. In the published literature, ${ }^{1,2}$ fully segmented nonincarcerated hemivertebrae with normal growth plate are considered a more likely cause of progressing deformity, particularly when associated with contralateral bone bar. However, even semi-segmented hemivertebrae can cause significant deformity. Hemivertebrae location is significant in deformity progression risk, as in congenital scoliosis by hemivertebrae of the mobile spine at the lumbar, thoracolumbar, cervical, and thoracocervical level levels, and it is more likely to progress than hemivertebrae at the thoracic level, where the thoracic cage could limit deformity progression. In congenital kyphosis, high thoracic location is associated with the worst progression risk. ${ }^{3}$

Because bracing is ineffective in limiting deformity progression and with potential risks of neurologic impairment in the case of kyphosis and kyphoscoliosis, ${ }^{4-6}$ surgery is proposed to treat progressive deformity. From 2006, our group has treated progressive spine deformity caused by hemivertebra at the lumbar, thoracic, and thoracolumbar levels using a total hemivertebra resection through a posterior-only approach with transpedicular instrumentation to obtain correction. Our technique is similar to that which has been described by 
Ruf and Harms. ${ }^{7,8}$ Prior to 2006, we used to treat congenital deformities with an anterior-posterior combined approach resection in a 2-stage procedure.

Our purpose was to analyze and present the results of one-time posterior hemivertebra resection and spinal arthrodesis for young patients with congenital scoliosis at a midterm follow-up.

\section{MATERIALS AND METHODS}

The current study is the continuation of our previous article, in which we described our cases operated on from 2006 to $2011 .{ }^{9}$ In that work, we described our surgical procedure as well as preoperative and follow-up imaging control program.

\section{Patients}

From 2006 to 2019, we operated on 82 patients with congenital vertebral deformities (62 scoliosis and 20 kyphoscoliosis), performing hemivertebra resection and instrumented arthrodesis with pedicle screws through a one-time posterior approach. All patients had curve progression with deformity worsening (mean scoliosis worsening $9^{\circ}$ Cobb per year, range $5^{\circ}-25^{\circ}$; mean kyphosis worsening $15^{\circ} \mathrm{Cobb}$ per year, range $8^{\circ}-$ $30^{\circ}$ ). All patients were operated on by the same senior surgeon (M.C.).

The mean age at the time of surgery was 8.6 years (18 months-16.2 years), and 22 patients were younger than 10 years (mean age 5.5 years, range 18 months -9.5 years).

In surgical procedures until 2011, we did not use intraoperative neurophysiologic monitoring (IONM), which was used as standard procedure in operations after 2011. After stabilization, patients were immobilized in a cast and/or a brace for 3 to 5 months. All patients were evaluated using standard x-ray images of the spine (posterior-anterior and lateral) at 1, 3, 6, and 12 months after surgery, and then every 12 months.

\section{Surgical Techniques and Approaches}

In patients younger than 10 years without secondary structured curves, we resected hemivertebra and fused the 2 adjacent vertebrae. When necessary, 1 or 2 additional segments were included into fusion, depending on curve length. In these patients, we never used cages to fill the gap left by resection because we never had difficulties in completely closing the gap acting on instrumentation rods, probably due to the greater curve plasticity of these patients.
When the curves were spared on more levels, particularly in patients older than 10 years with greater deformity curves and sometimes secondary structured curves, we resected the hemivertebra and used longer instrumented fusion to cover more spanned and structured curves to obtain complete deformity correction. In a case of congenital kyphoscoliosis in a boy aged about 13.5-year-old, due to L2-L3 hemivertebra, we performed hemivertebra resection, L2-L3 anterior interbody fusion using a titanium cage with autologous bone morselized graft, inserted by posterior approach, and T12-L4 posterior instrumented arthrodesis (Figure 1).

We operated on 1 case with hemivertebra resection at multiple levels: a 6-year-old and 7-month-old boy presented with L2-L3 right, partially free hemivertebra, T1-T2 right hemivertebra, and $\mathrm{C} 6-\mathrm{C} 7$ hemivertebra. Based on our observations, the worst curve progression was sustained by lumbar hemivertebra, and we at first resected L2-L3 hemivertebra. The patient had complex anatomy deformity in the lumbar spine, a common hiatus for L2-L3 nerve, and the only pedicle available for screw insertion below L2 was at hemivertebra level. L2-L4 posterior instrumented arthrodesis was performed closing the hemivertebra resection gap. Thirty months after the first surgery, the patient was treated by thoracic hemivertebra resection and $\mathrm{T} 1-\mathrm{T} 3$ posterior instrumented arthrodesis. The parents refused further treatment by cervical hemivertebra resection. Thus, the patient was treated by anterior-posterior combined approach to correct the cervical deformity. At 9-year follow-up from first intervention and 4-year and 3-month follow-up from second surgery, the patient has no loss of correction or lumbar or thoracic curves progression, has maintained a good functional and cosmetic result, and is actively involved in sports (Figure 2).

\section{RESULTS}

Mean follow-up was 9.6 years (range 1.2-12.8 years). Mean preoperative kyphosis curve was $75^{\circ} \mathrm{Cobb}$ (range $65^{\circ}-88^{\circ} \mathrm{Cobb}$ ); mean preoperative scoliosis curve was $44^{\circ}$ (range $26^{\circ}-60^{\circ}$ ); and mean segmental kyphosis value was $25^{\circ} \mathrm{Cobb}$ (range $10^{\circ}-60^{\circ}$ ).

Mean kyphosis curve after surgery was reduced to $20^{\circ}$ Cobb (range $10^{\circ}-30^{\circ} \mathrm{Cobb}$ ), and mean scoliosis curve was reduced to $11^{\circ} \mathrm{Cobb}$ (range $9^{\circ}-20^{\circ} \mathrm{Cobb}$ ). Mean segmental value at follow-up was $-1.9^{\circ} \mathrm{Cobb}$ (range $-5^{\circ}$ to $0^{\circ} \mathrm{Cobb}$ ). Mean blood loss was $370 \mathrm{~mL}$ (range 200-735 $\mathrm{mL}$ ) and mean operative time was 227 min (range 119-325 min).

We had no major complications after surgery (severe neurologic impairment, infection, instrumentation 


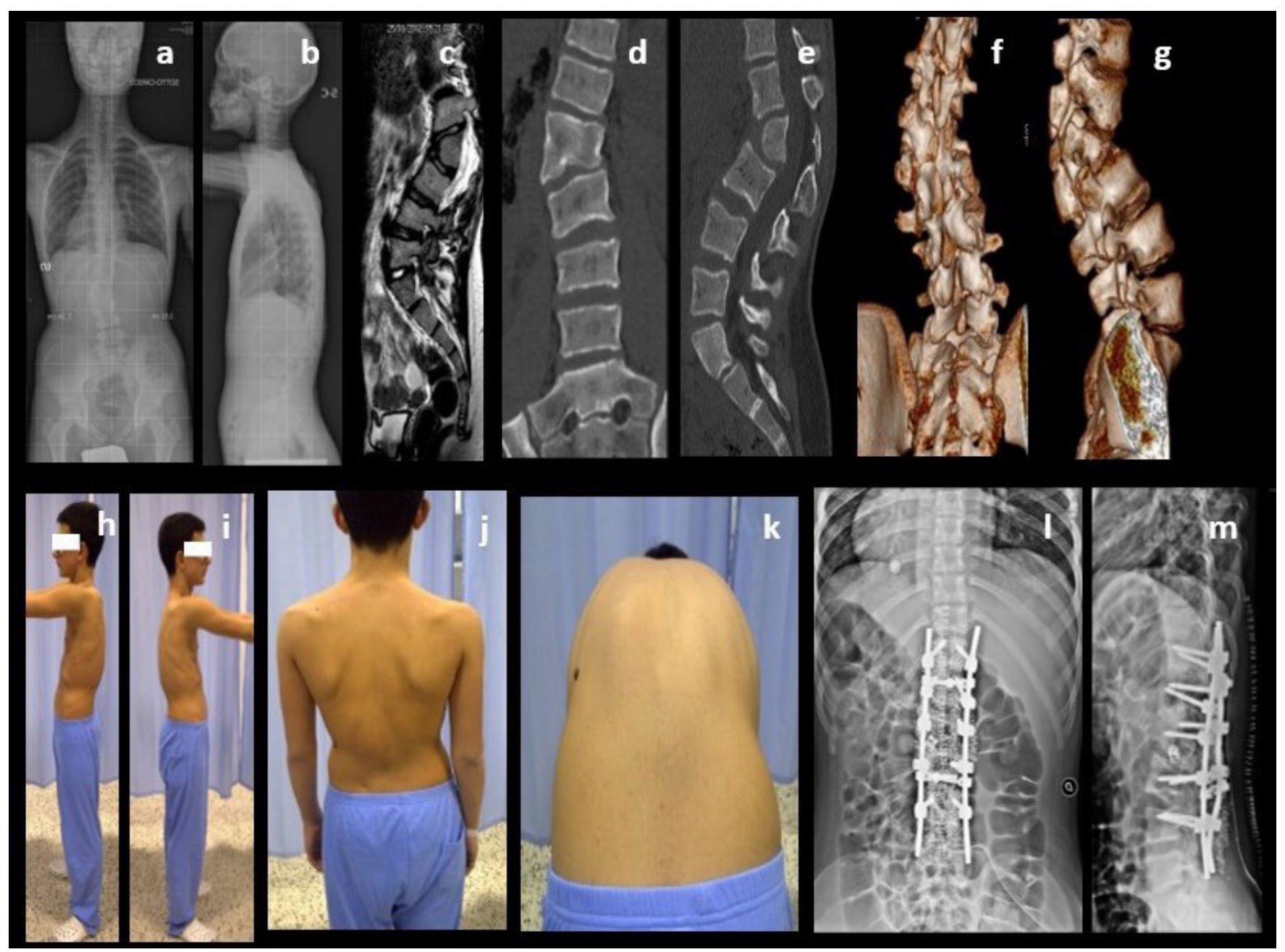

Figure 1. Preoperative $x$-ray images (a, b), magnetic resonance images (c), computed tomographic (CT) images (d, e), three-dimensional CT images (f, g), and clinical presentation $(\mathrm{h}-\mathrm{k})$ of the patient. Postoperative $\mathrm{x}$-ray images $(\mathrm{l}, \mathrm{m})$.

failure, visceral or vascular iatrogenic injury, or massive hemorrhage). Despite not using IONM before 2011, as it was not available in our institution before that date, we did not have any serious nervous impairment in patients operated on before 2011, nor in patients operated on after that date with IONM aid.

In 1 patient, we observed transitory paresthesia in the lower left leg after surgery. This patient presented a neurologic anatomy abnormality, double spinal root in a single hiatus at L5 level. This neurologic symptom was completely resolved 2 months after surgery; she is free of neurologic impairment and has a normal gait at 6.4 years follow-up.

In another case, we had pedicle fracture during pedicle screw insertion. We inserted a pedicle screw 1 level above: no implant failure or loss of correction was observed at 7.3 years follow-up; the patient had no pain or deformity, and fusion at the treated levels was complete (Figure 3).
We never had any cases of instrumentation mobilization or nonunion at follow-up. We experienced 6 cases (8\%) of deformity progression: 1 case of proximal junction kyphosis in congenital scoliosis. The patient was treated at 8.3 years by L2-L3 hemivertebra resection and L2-L4 pedicle screw instrumentation, with good scoliosis correction, but 6 months after surgery he developed proximal junction kyphosis over instrumentation, while before surgery patient's sagittal profile was normal. Kyphosis has been controlled by bracing (Figure 4). The other 4 cases were scoliosis progression after hemivertebra resection and short fusion 1 level above and below resection, with pedicle screw instrumentation. In every case, surgery obtained an immediate postoperative complete correction in the hemivertebra area, with good correction of scoliosis deformities, but at an average of 6 months follow-up scoliosis curve progressed to maximum $15^{\circ} \mathrm{Cobb}$, showing what we called "memory of deformity" (Figure 5). X-ray images 


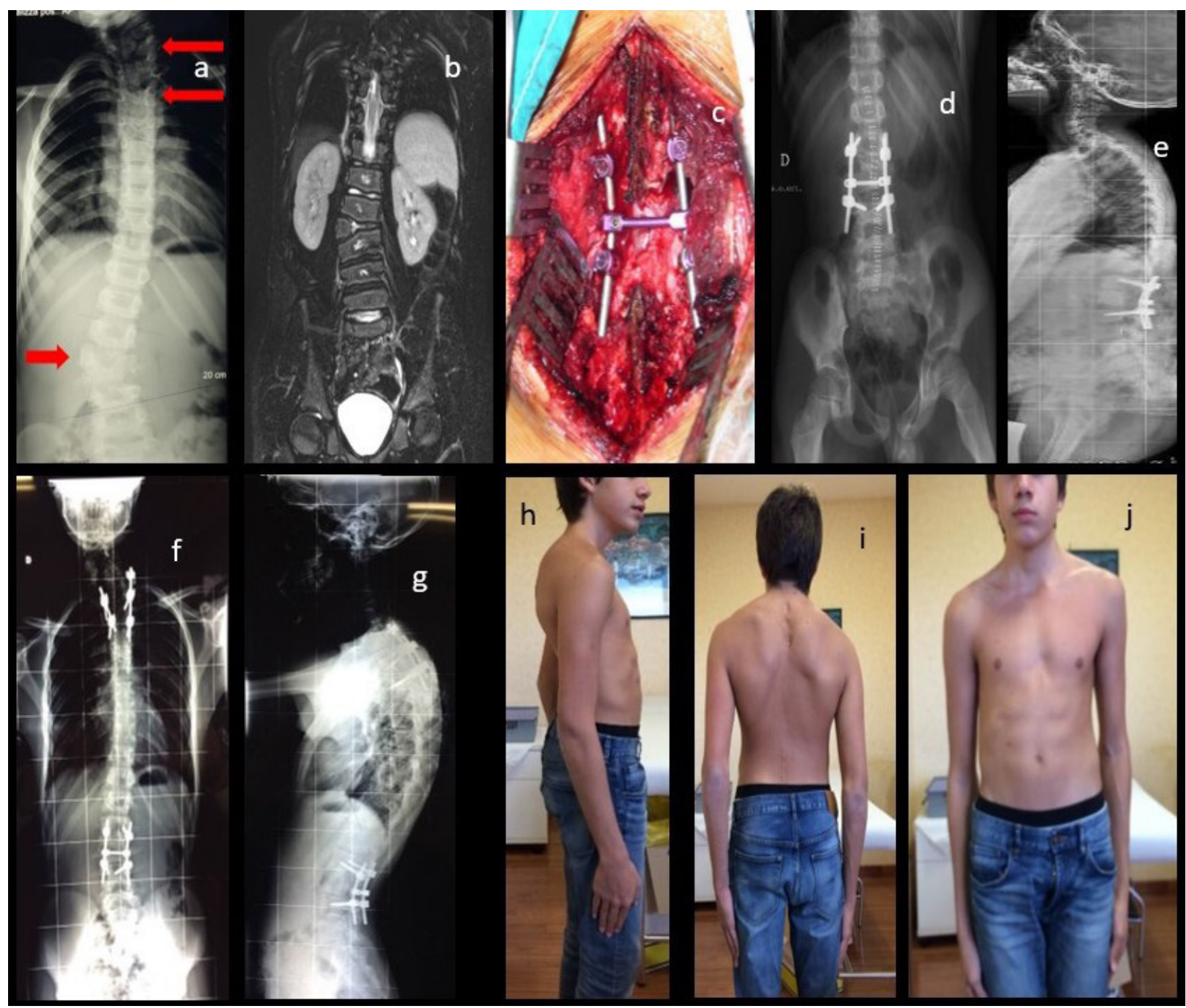

Figure 2. Preoperative x-ray image (a) showing 3 hemivertebras (arrows); preoperative magnetic resonance image (b); intraoperative picture showing lumbar instrumentation (c); postoperative x-ray images (d, e); postoperative x-ray images after the second procedure (f, g); and clinical pictures at latest follow-up 8.8 years after lumbar procedure and 4.7 years after thoracic hemivertebra resection $(\mathrm{h}-\mathrm{j})$.

at follow-up showed complete hemivertebra removal, confirming that the curve is a developing deformity related to a previous deformity but not a relapse of the original scoliosis due to incomplete hemivertebra resection, with progressive growth of the residual malformed segment. In 2 cases, the new deformity has been treated by bracing, while in the remaining cases we are observing curve development without treatment, as further curve progression is not appreciable.

\section{DISCUSSION}

The severity of congenital scoliosis or kyphoscoliosis at the time of surgery and the progression behavior of the deformity, or the expected progression of deformity leads to surgical indication. Surgery goals are achieving a straight spine on frontal view with a physiologic sagittal profile with as short a fusion segment as possible. ${ }^{10}$ Natural history of progressive deformity is the development of secondary curves in scoliosis, with the necessity of long fusion segments. Kyphosis and kyphoscoliosis progression can result in severe nervous impairment. Moreover, correction of rigid structured curves is associated with a higher rate of complications. ${ }^{10}$ Early correction in young children requires a less invasive approach and a short and sufficient rigid instrumentation. A posterior-only hemivertebra resection is the result of applying to congenital deformities, techniques developed in the past decades to treat 


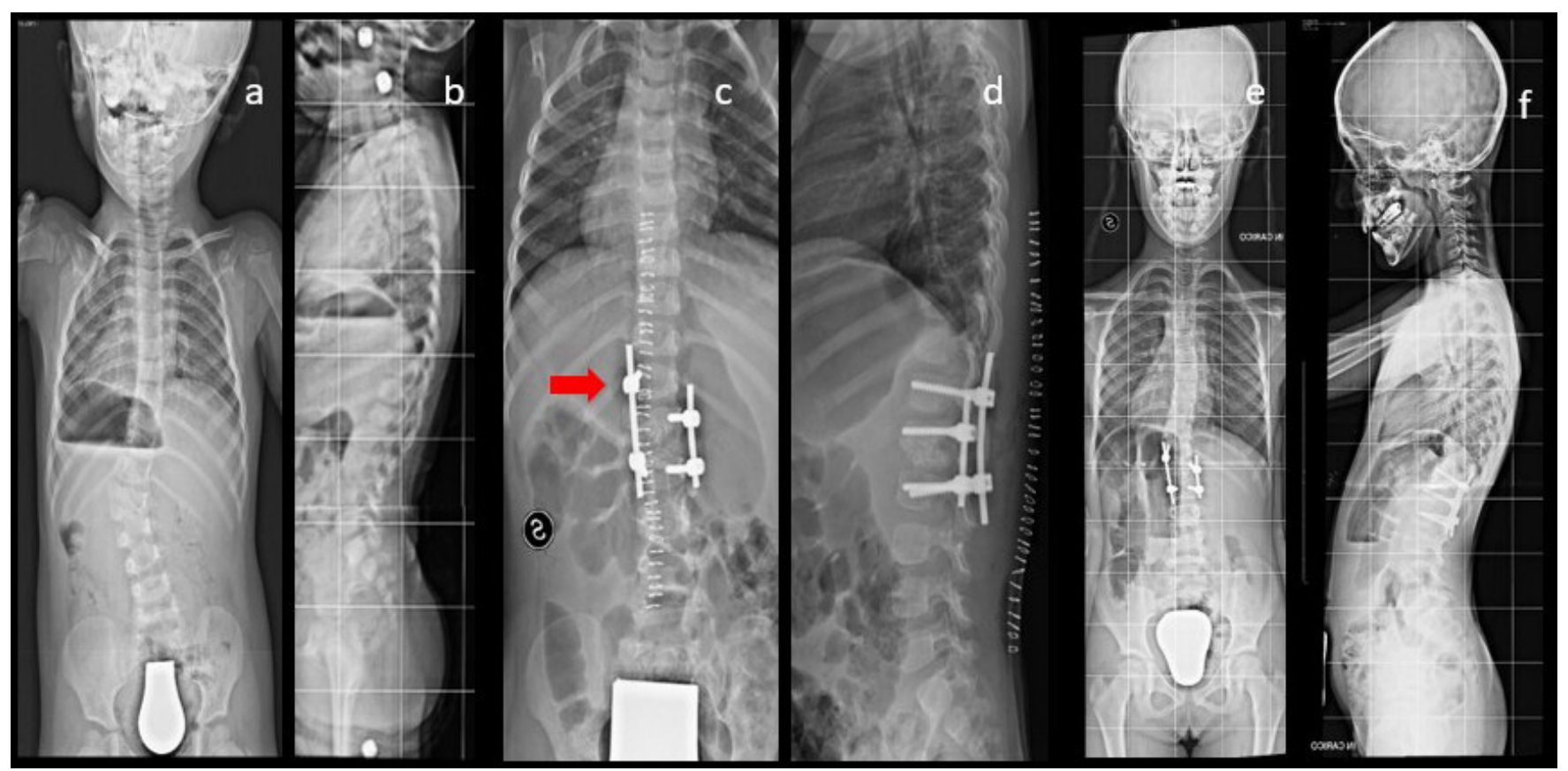

Figure 3. Preoperative x-ray images of a 25-month-old patient (a, b); postoperative x-ray images showing T12 left pedicle screws (arrow) after rupture of L1 left pedicle (c, d); and x-ray images at 7.3 years follow-up (e, f) showing no loss of correction.

pathologies of the anterior part of the spine by posterior approach. Rigid instrumentation is applied to obtain complete deformity correction, by closing the gap left by hemivertebra resection, with the use of compression on the convex side associated with distraction in curve concavity. Pedicle screws are better suited to transmit correction forces to vertebral bodies of a young child's spine than other instrumentation systems and are well tolerated even in our youngest patients, as the pedicles are able to withstand the compression forces needed to close the gap left by hemivertebra resection. In contrast with results reported by other authors, ${ }^{3,7,11,12}$ we had no risk of complications related to construct or implant, even in patients under 5 years and with 2-level fusions.

In our series, the only patient with intraoperatory pedicle fracture was a 25 -month-old child. We rose the pedicle screw 1 level on 1 side, without impairment of instrumentation stability or of arthrodesis formation at

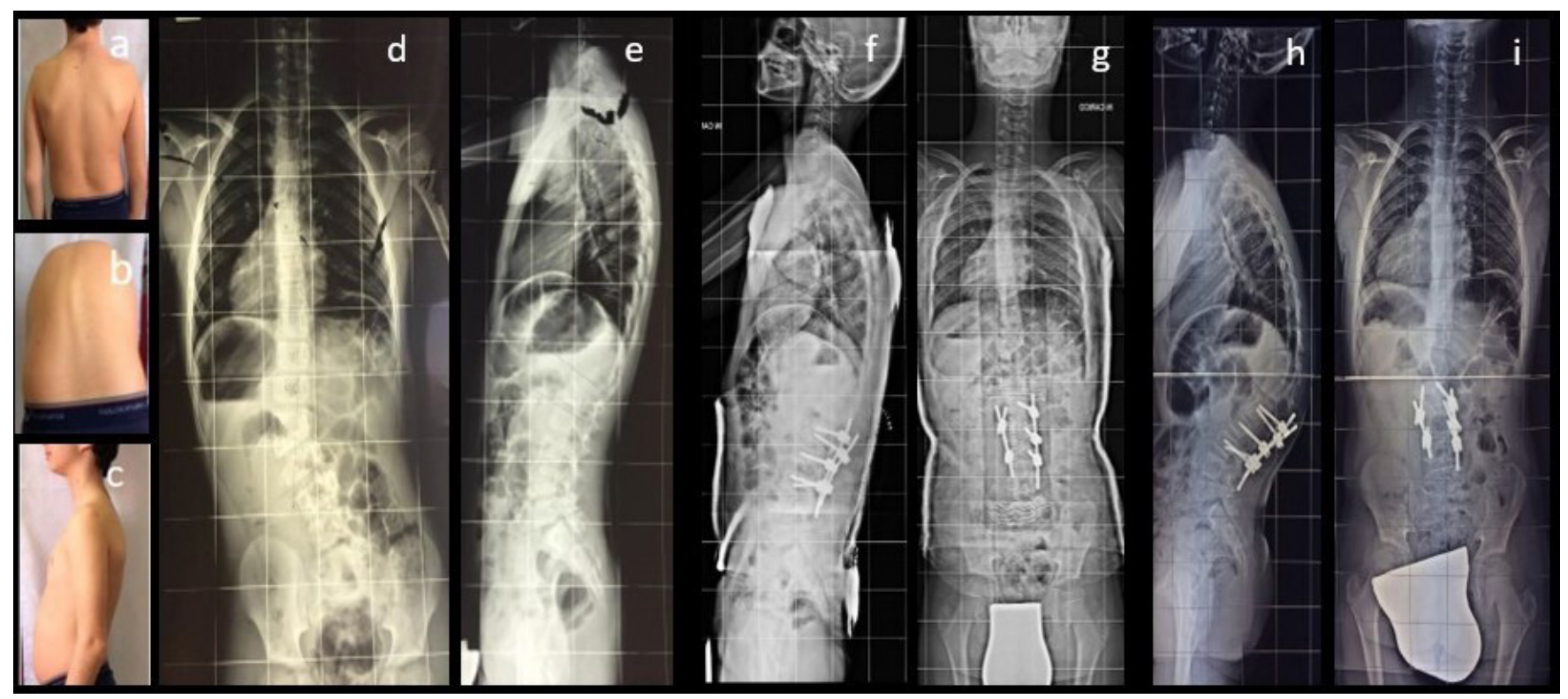

Figure 4. Clinical presentation $(a-c)$ and preoperative $x$-ray images (d, e); postoperative x-ray images with cast (f, g); and 6 months follow-up x-ray images showing proximal junctional kyphosis (h, i). 


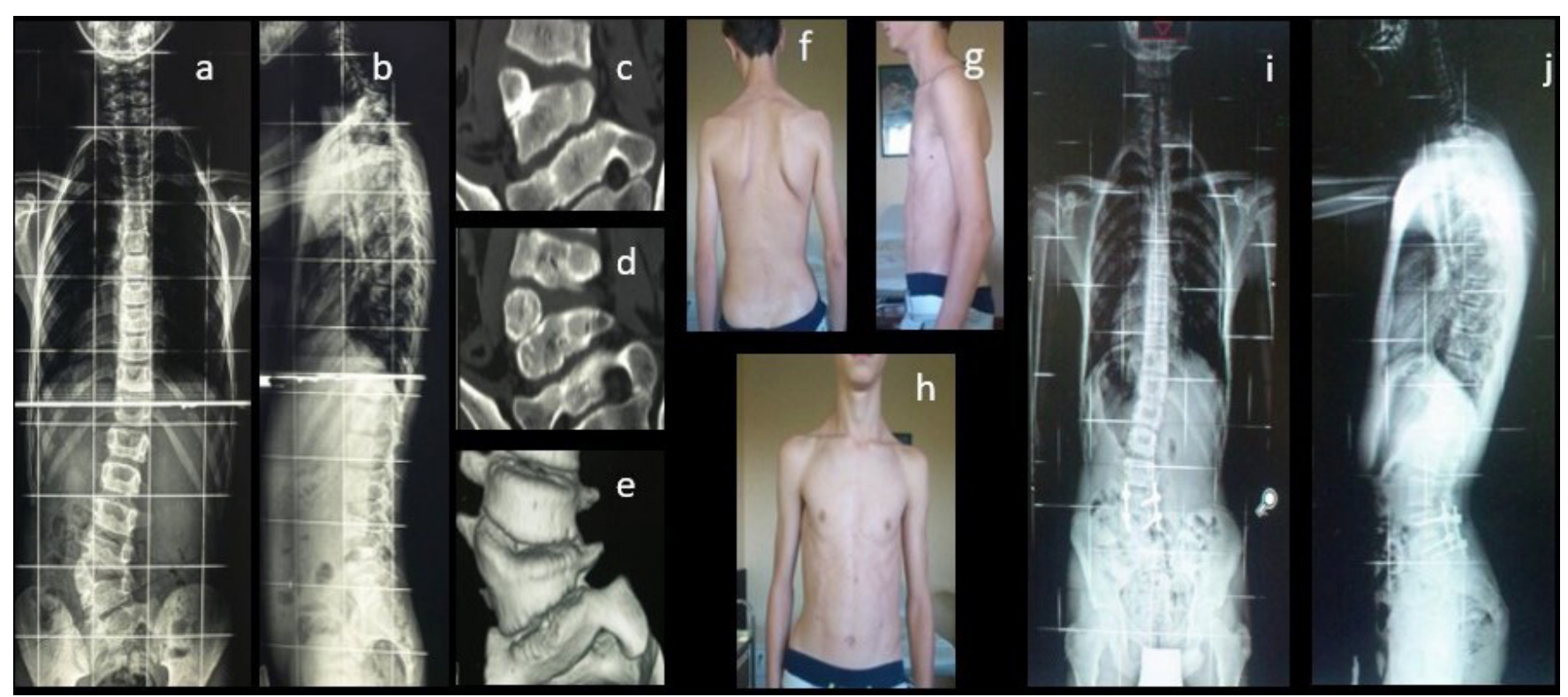

Figure 5. Preoperative x-ray images (a, b), computed tomographic (CT) images (c, d), and three-dimensional CT image (e); and clinical (f-h) and radiologic (i, j) findings at 8 months follow-up, showing small upper scoliotic curve development and slight cosmetic impairment.

follow-up (Figure 3). We believe that fracture is more likely due to surgical technique defect and not to intrinsic pedicle fragility. We operated on 13 patients under 6 years and one 6-year-old without any other pedicle fracture, secondary instrumentation mobilization or arthrodesis impairment at follow-up.

At follow-up x-ray evaluations, the rate of suboptimal positioning of pedicle screws was 5\% due to smaller anatomy, but without nervous complications, implant loosening or loss of correction.

All patients were braced for a mean time of 3 months after surgery. We think that the relatively long period of bracing of our patients allows the maturation of a complete arthrodesis, even in presence of suboptimal screw positioning, and it is the reason for no secondary implant loosening or nonunion in our cases. The spinal canal is fully formed at 1 year and pedicle screws inserted in very young children do not cause spinal canal growth impairment, as experimental studies and clinical experience have shown. ${ }^{13}$

In a growing spine, fusion segment is kept as short as possible $^{8}$ to minimize the compromise of normal spine development, even if short fusion may increase the risk of new deformity after surgery. We experienced 6 cases of curve progression on frontal or sagittal plane after surgery: 1 case of junctional kyphosis after L2-L3 partially fused hemivertebra resection in congenital scoliosis (Figure 4) and 4 cases of scoliosis curve progression.

As posterior hemivertebra resection and short fusion became a more widely applied treatment in the correction of progressive congenital scoliosis, postoperative new deformity has been reported as one of the most common complications of this surgery. ${ }^{14}$ Deformity progression has a huge impact on initial correction and may lead to revision surgery. Wang et $\mathrm{al}^{15}$ reported that $18.9 \%$ of congenital scoliosis patient with posterior hemivertebra resection and short fusion $(7$ in a series of 37) developed more than $10^{\circ}$ kyphosis above the top of the instrumentation in a mean 4 years 6-month follow-up period. These authors analyzed 2 factors that possibly caused proximal junction kyphosis: the first being that the misplaced screws in upper instrumented vertebra may have disturbed the normal growth of the endplate, and second, the damage that may have been caused in the facet capsules and interspinous ligament during exposure. Shi et al, ${ }^{16}$ Ruf et al, ${ }^{17}$ and Zhang et $\mathrm{al}^{18}$ reported cases of spine deformity due to hemivertebra, which underwent revision surgery because of deformity progression after hemivertebra resection and fusion. At follow-up, they did not find any incomplete resection of hemivertebra, instrumentation failure, or nonunion development as a cause of deformity progression. Junction kyphosis and scoliosis progression after surgery develop at hemivertebra resection level or at surgical fusion area. Yang et $\mathrm{al}^{14}$ in 2016 reported the development of an " $\mathrm{S}$ "-shaped scoliosis in 9 patients in a case mix of 128 congenital scoliosis, operated on using hemivertebra resection and short segment fusion. The authors defined this curve as "coronal decompensation."

The apical vertebra of the new curves was at least 2 levels far from the upper or lower instrumented vertebra, 
differing from previously reported add-on deformities. Scoliosis developed 3-6 months after hemivertebra resection, and at follow-up, there was neither incomplete hemivertebra resection nor instrumentation failure. The patients were treated with bracing, and 4 underwent revision surgery.

Yang et $\mathrm{al}^{14}$ theorize that this deformity develops from slight and compensatory secondary scoliosis, present in the spine before surgery, and that the scoliosis after surgery develops similarly to idiopathic scoliosis. According to Yang et al's theory, we think that these scoliosis curves are similar to idiopathic scoliosis curves, but that they have a "memory relation" with primary deformity, meaning that these curves would have developed at a wider angle if hemivertebra had not been resected and if hemivertebra persistence would have sustained the scoliosis curves.

As the pathogenetic mechanism and predictive factors of deformity progression after hemivertebra resection and short fusion are still unclear, it is particularly difficult to understand whether progression is due to technical error (iatrogenic damage of tissues, too short fusion area choice), or to subsistent pathology.

In a group of 179 patients younger than 5 years treated for congenital scoliosis by posterior-only hemivertebra resection and short fusion with minimum 2 years follow-up, Li et al ${ }^{19}$ found postoperative coronal decompensation in 18 patients $(10.1 \%)$. They identified preoperative lowest instrumented vertebra translation $\geq 15.1 \mathrm{~mm}$ and postoperative lowest instrumented vertebra disc angle $\geq 5.5^{\circ}$ as adverse predictive factors for postoperative coronal decompensation. According to $\mathrm{Li}$ et al, this complication mainly occurred within 3 to 6 months after surgery. The authors stated that if a significant wedging of the lower instrumented vertebra disc occurs after surgery, the spine could experience an unbalanced loading between the 2 sides caudally to lower instrumented vertebra, according to HueterVolkmann law, resulting in asymmetrical spinal growth.

It is possible that correction maneuvres on hemivertebra resection gap can trigger unbalanced stress on disc of vertebras above and below resection area, causing asymmetrical spinal growth and coronal decompensation.

In our patients, we experienced a relatively low rate of deformity progression ( $8 \%$ ); even if the sample is too small for any statistical significance, it shows that short fusion following posterior hemivertebra resection is still the first choice for patients with congenital scoliosis due to hemivertebra. In our experience, eventual proximal junctional kyphosis or coronal decompensation deformities in young children after hemivertebra resection can be effectively treated by bracing, as Li et al also have described. ${ }^{19}$

However, secondary curves can become more structured and stiff with growth.

In cases of sagittal or coronal decompensation where an initially flexible adding curve develops in a structural one, extension of instrumented arthrodesis might be a solution to obtain definitive correction.

\section{CONCLUSIONS}

The revision of our cases some years after our first report confirms the effectiveness of posterior-only approach hemivertebra resection in congenital spine deformities treatment. Single-stage posterior hemivertebra resection and short fusion have become the mainstream treatment for progressive congenital scoliosis. ${ }^{9,20}$ Early or even prophylactic hemivertebra resection was recommended to stop the development of local deformity and to prevent longer fusion extending to the secondary structural curves. ${ }^{20}$ Modern transpedicular instrumentation could provide the short and sufficiently rigid stabilization in such patients, thereby avoiding wider distractions of spinal growth and mobility. ${ }^{17}$

Posterior approach interventions with pedicle screws instrumentation are less invasive than anterior-posterior combined approach interventions and are well tolerated, even by very young patients. Pedicle screws achieve important corrections that can be maintained by filling the resulting gaps with bone obtained by resection. If intervention is performed early, before secondary curves are structured, the arthrodesis area can be limited to 1 vertebra proximal and 1 distal to the hemivertebra.

Proximal junctional kyphosis and coronal decompensation are possible complications, and the real causes of curve progression after a complete hemivertebra resection and instrumentation are still unclear; otherwise, incidence of curve progression is relatively low, and most cases have been successfully treated by bracing. Future studies are due to observe long-term outcome of cases of secondary curves after hemivertebra resection.

We believe that posterior approach procedures obtain excellent deformity correction in both the frontal and sagittal plane, and optimal stability with low risk of major complications.

\section{REFERENCES}

1. McMaster MJ, David CV. Hemivertebra as a cause of scoliosis. A study of 104 patients. J Bone Joint Surg Br. 1986;68-B(4):588-595. doi:10.1302/0301-620X.68B4.3733836 
2. McMaster MJ, Ohtsuka K. The natural history of congenital scoliosis. A study of two hundred and fifty-one patients. The Journal of Bone \& Joint Surgery. 1982;64(8):1128-1147. doi:10.2106/00004623-198264080-00003

3. Ansari SF, Rodgers RB, Fulkerson DH. Dorsal midline hemivertebra at the lumbosacral junction: report of 2 cases. J Neurosurg Spine. 2015;22(1):84-89. doi:10.3171/2014.9.SPINE1411

4. Williams F, McCall IW, O'Brien JP, Park WM. Severe kyphosis due to congenital dorsal hemivertebra. Clin Radiol. 1982;33(4):445-452. doi:10.1016/s0009-9260(82)80315-2

5. Winter RB. Congenital kyphosis. Clin Orthop Relat Res. 1977;amp;NA(128):26. doi:10.1097/00003086-197710000-00005

6. Winter RB, Moe JH, Wang JF. Congenital kyphosis. Its natural history and treatment as observed in a study of one hundred and thirty patients. J Bone Joint Surg Am. 1973;55(2):223-256.

7. Ruf M, Harms J. Posterior hemivertebra resection with transpedicular instrumentation: early correction in children aged 1 to 6 years. Spine. 2003;28(18):2132-2138. doi:10.1097/01. BRS.0000084627.57308.4A

8. Ruf M, Harms J. Hemivertebra resection by a posterior approach. Spine. 2002;27(10):1116-1123. doi:10.1097/00007632200205150-00020

9. Crostelli M, Mazza O, Mariani M. Posterior approach lumbar and thoracolumbar hemivertebra resection in congenital scoliosis in children under 10 years of age: results with 3 years mean follow up. Eur Spine J. 2014;23(1):209-215. doi:10.1007/s00586-013-2933-z

10. Jalanko T, Rintala R, Puisto V, Helenius I. Hemivertebra resection for congenital scoliosis in young children: comparison of clinical, radiographic, and health-related quality of life outcomes between the anteroposterior and posterolateral approaches. Spine. 2011;36(1):41-49. doi:10.1097/BRS.0b013e3181ccafd4

11. Guo J, Zhang J, Wang S, et al. Risk factors for construct/ implant related complications following primary posterior hemivertebra resection: study on 116 cases with more than 2 years' follow-up in one medical center. BMC Musculoskelet Disord. 2016;17(1):380. doi:10.1186/s12891-016-1229-y

12. Zhu X, Wei X, Chen J, et al. Posterior hemivertebra resection and monosegmental fusion in the treatment of congenital scoliosis. Ann R Coll Surg Engl. 2014;96(1):41-44. doi:10.1308/003588 414X13824511650173

13. Jeszensky D. Morphological changes of the spinal canal after placement of pedicle screws in newborn pigs. Scoliosis Research Society Annual Meeting. 2000.

14. Yang X, Song Y, Liu L, et al. Emerging S-shaped curves in congenital scoliosis after hemivertebra resection and short segmental fusion. Spine J. 2016;16(10):1214-1220. doi:10.1016/j. spinee.2016.06.006

15. Wang S, Zhang J, Qiu G, Li S, Yu B, Weng X. Posterior hemivertebra resection with bisegmental fusion for congenital scoliosis: more than 3 year outcomes and analysis of unanticipated surgeries. Eur Spine J. 2013;22(2):387-393. doi:10.1007/s00586012-2577-4

16. Shi Z, Li Q, Cai B, et al. Causes of the failure and the revision methods for congenital scoliosis due to hemivertebra. Congenit Anom. 2015;55(3):150-154. doi:10.1111/cga.12107

17. Ruf M, Jensen R, Letko L, Harms J. Hemivertebra resection and osteotomies in congenital spine deformity. Spine. 2009;34(17):1791-1799. doi:10.1097/BRS.0b013e3181ab6290

18. Zhang J, Shengru W, Qiu G, Yu B, Yipeng W, Luk KDK. The efficacy and complications of posterior hemivertebra resection. Eur Spine J. 2011;20(10):1692-1702. doi:10.1007/s00586-0111710-0

19. Li S, Chen Z-H, Qiu Y, et al. Coronal decompensation after posterior-only thoracolumbar hemivertebra resection and short fusion in young children with congenital scoliosis. Spine. 2018;43(9):654-660. doi:10.1097/BRS.0000000000002383

20. Chang D-G, Suk S-I, Kim J-H, Ha K-Y, Na K-H, Lee J-H. Surgical outcomes by age at the time of surgery in the treatment of congenital scoliosis in children under age 10 years. Spine J. 2015;15(8):1783-1795. doi:10.1016/j.spinee.2015.04.009

Funding: The author(s) received no financial support for the research, authorship, and/or publication of this article.

Declaration of Conflicting Interests: The authors report no conflicts of interest related to this article.

Dedication: In loving memory of our beloved and esteemed friend and colleague Massimo Mariani, whose contribution was fundamental for the drafting of this work, who sadly passed away fulfilling his profession during the present pandemic.

Corresponding Author: Carlo Iorio, Spine Surgery Unit, Bambino Gesù Children's Hospital, Piazza Sant'Onofrio, 400165 Rome, Italy; carlo.iorio@ opbg.net

Published 15 February 2022

This manuscript is generously published free of charge by ISASS, the International Society for the Advancement of Spine Surgery. Copyright (C) 2022 ISASS. To see more or order reprints or permissions, see http:// ijssurgery.com. 